\title{
Ultrasound elastography in the evaluation of peripheral neuropathies: a systematic review of the literature
}

\author{
Jakub Zakrzewski ${ }^{1 \mathrm{~A}, \mathrm{~B}, \mathrm{D}, \mathrm{E}, \mathrm{F}}$, Katarzyna Zakrzewska ${ }^{2 \mathrm{~B}, \mathrm{~F}}$, Katarzyna Pluta ${ }^{1 \mathrm{~B}, \mathrm{~F}}$, Oleg Nowak ${ }^{1 \mathrm{~B}, \mathrm{~F}}$, \\ Anna Miłoszewska-Paluch ${ }^{3 B, F}$ \\ 'Department of Radiology, Gruca Orthopedic and Trauma Teaching Hospital, Center of Postgraduate Medical Education, Otwock, Poland \\ 2Department of Dermatology, Military Institute of Medicine, Warsaw, Poland \\ ${ }^{3}$ Department of Pediatric Dentistry, The Infant Jesus Teaching Hospital in Warsaw, Poland
}

\section{Abstract}

\begin{abstract}
Peripheral neuropathy is associated with an increase in intraneural pressure, and hence ultrasound elastography seems to be an ideal method to detect early stages of this condition based on changes in the affected nerve stiffness. The aim of this systematic review was to analyse the applicability of strain elastography (SE) and shear wave elastography (SWE) in the evaluation of peripheral nerves in patients with neuropathy of various aetiologies. Published evidence shows clearly that ultrasound elastography can accurately diagnose many types of peripheral neuropathies (carpal tunnel syndrome and other entrapment neuropathies, diabetic peripheral neuropathy and peripheral neuropathy associated with other systemic diseases), sometimes at the stages at which the condition is still asymptomatic. However, it is still unclear whether elastographic changes within the nerves precede functional anomalies detectable on nerve conduction studies. Also, relatively little is known about the relationship between the stiffness of peripheral nerves and the severity of peripheral neuropathy and its underlying condition. Based on the reproducibility data, SWE seems to be superior to SE. Nevertheless, the sources of heterogeneity in the peripheral nerve stiffness in healthy persons need to be identified, and the sets of reference values for specific peripheral nerves need to be determined. Finally, the potential confounding effect of hardening artefacts, such as bones, on the stiffness of peripheral nerves needs to be verified. After addressing all these issues, elastographic evaluation of peripheral nerve stiffness might become a reliable, easily accessible, and convenient diagnostic test performed routinely in patients with various peripheral neuropathies.
\end{abstract}

Key words: elastography, carpal tunnel syndrome, entrapment neuropathy, peripheral diabetic neuropathy, peripheral neuropathy.

\section{Introduction}

The term 'peripheral neuropathy' refers to a condition affecting at least one peripheral nerve and contributing to impairment of its motor, sensory, and/or autonomic function. Common causes of neuropathy include systemic diseases, nerve entrapment and/or ischaemia, inflammation (neuritis), traumatic injuries, viral infections, vitamin deficiencies, and some medications. In most cases, neuropathy is associated with an increase in intraneural pressure, resultant nerve oedema, compression of supplying blood vessels (vasa nervorum), and ischaemia. If prolonged, this will eventually lead to demyelination, axonal atrophy with secondary fibrosis, and functional deterioration of the nerve [1].

Because typically there is a lag phase between the onset of nerve degeneration and clinically evident dysfunction, diagnostic tests capable of detecting peripheral neuropathy at subclinical stages are of utmost importance. Depending on its location, function of the affected nerve can be controlled by nerve conduction studies (NCS), and magnetic resonance imaging (MRI) was shown to visualise

Correspondence address:

Dr. Jakub Zakrzewski, Department of Radiology, Gruca Orthopedic and Trauma Teaching Hospital, Center of Postgraduate Medical Education, Otwock, Poland,

e-mail: jakub.a.zakrzewski@gmail.com

Authors' contribution:

A Study design · B Data collection · C Statistical analysis · D Data interpretation · E Manuscript preparation · F Literature search · G Funds collection 
some early, preclinical alterations in peripheral nerve morphology. However, these tests are expensive, not widely available, and inconvenient; furthermore, they are either invasive (NCS) or require administration of contrast agent (MRI), and their sensitivity and specificity are far from optimum [2-5].

Considering its lack of invasiveness, better availability, and lower cost, conventional grey-scale ultrasound has been increasingly used as an accessory diagnostic test in patients with suspected peripheral neuropathies. Using conventional ultrasound, one can detect morphological changes in the affected peripheral nerve and quantify its cross-sectional area (CSA) [6,7]. However, as shown in this review, grey-scale ultrasound also has some limitations in the evaluation of peripheral nerves. These drawbacks can be overcome with ultrasound elastography, a relatively new diagnostic modality introduced in the 1990s. Elastography is an ultrasound technique to evaluate biological tissue stiffness [8]. The two most commonly used elastographic techniques are strain elastography (SE) and shear wave elastography (SWE). The former, older one is based on operator-induced compression with a transducer to evaluate tissue displacement and hence its elasticity/stiffness. The result is presented on a qualitative colour scale, with elastic, intermediate, and stiff tissues marked in red, green, and blue, respectively, or semi-quantitatively, as a strain/elasticity ratio between structures. In more recently introduced SWE, the velocity of a transducer-generated shear wave is measured across the tissue of interest and expressed as a quantitative parameter, in either kilopascals ( $\mathrm{kPa}$, as Young modulus) or metres per second (m/s) (Figures 1 and 2) [9].
Considering the previously mentioned pathophysiology of peripheral neuropathy with an increase in intraneural pressure and oedema, ultrasound elastography seems to be an ideal method to detect early stages of this condition based on changes in the affected nerve stiffness. Therefore, the aim of this systematic review was to analyse the applicability of SE and SWE in the evaluation of peripheral nerves in patients with neuropathy of various aetiologies.

\section{Material and methods}

A systematic search of the PubMed, Scopus, and Google Scholar was carried out. All published studies that analysed the applicability of ultrasound elastography, whether SE or SWE, in the evaluation of peripheral nerves and used the terms 'elastography' OR 'ultrasound elastography' OR 'strain elastography' OR 'shear wave elastography' AND 'neuropathy' OR 'peripheral neuropathy' OR 'diabetic neuropathy' OR 'neuritis' were identified and reviewed. The search limits were defined as 'English' (language), and 'the beginning of a given database through to 31 August 2019' (publication date). To be eligible for the systematic review, identified papers needed to compare the results of elastographic examination of peripheral nerves in patients with neuropathies of various origin and healthy controls. To provide a better insight into the applicability of ultrasound elastography in specific conditions, each chapter of this paper includes also information about a specific aetiology of peripheral neuropathy, other routinely used tests, and limitations thereof.

All records were identified from searches of the electronic databases, and duplicates were removed. Then, two

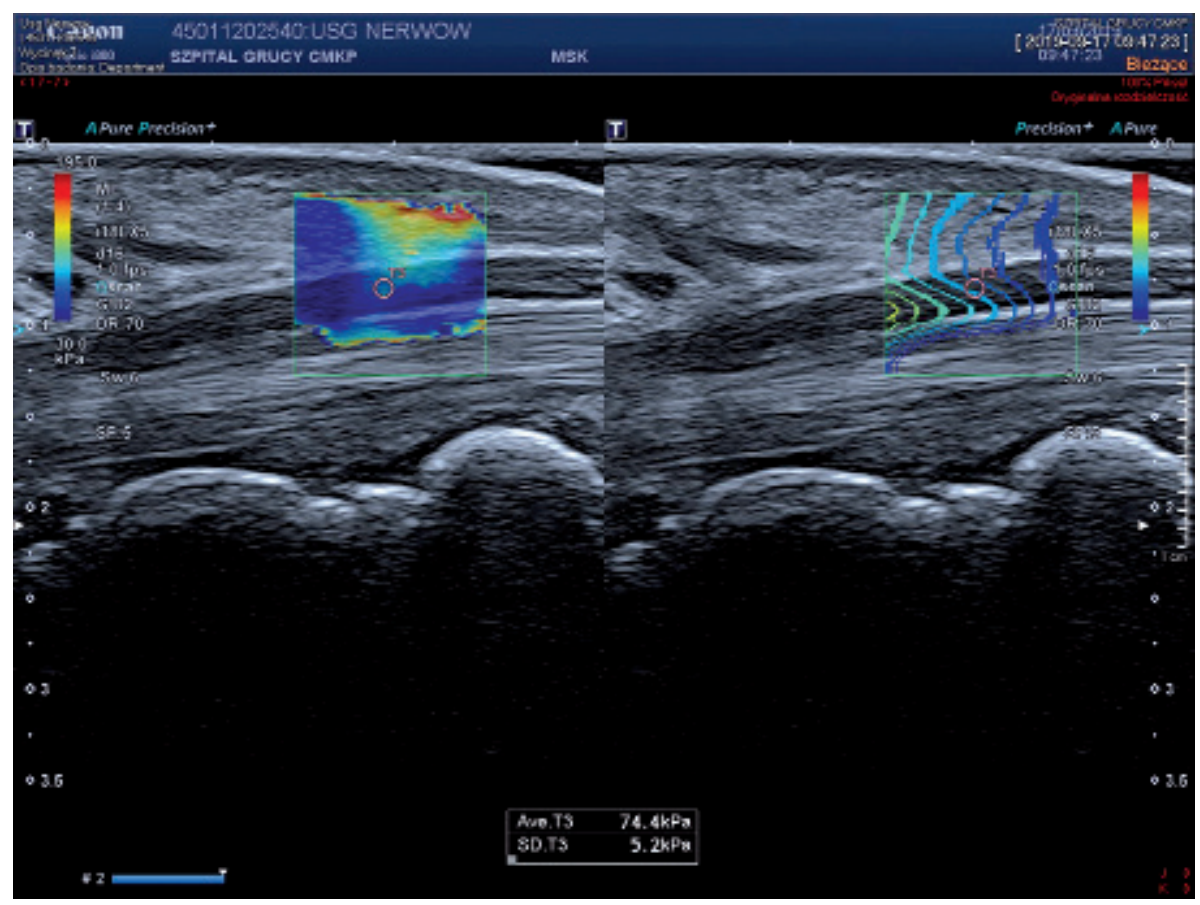

Figure 1. Shear wave elastography image of median nerve within the carpal tunnel in a 30-year-old man with no abnormalities in nerve conduction studies 


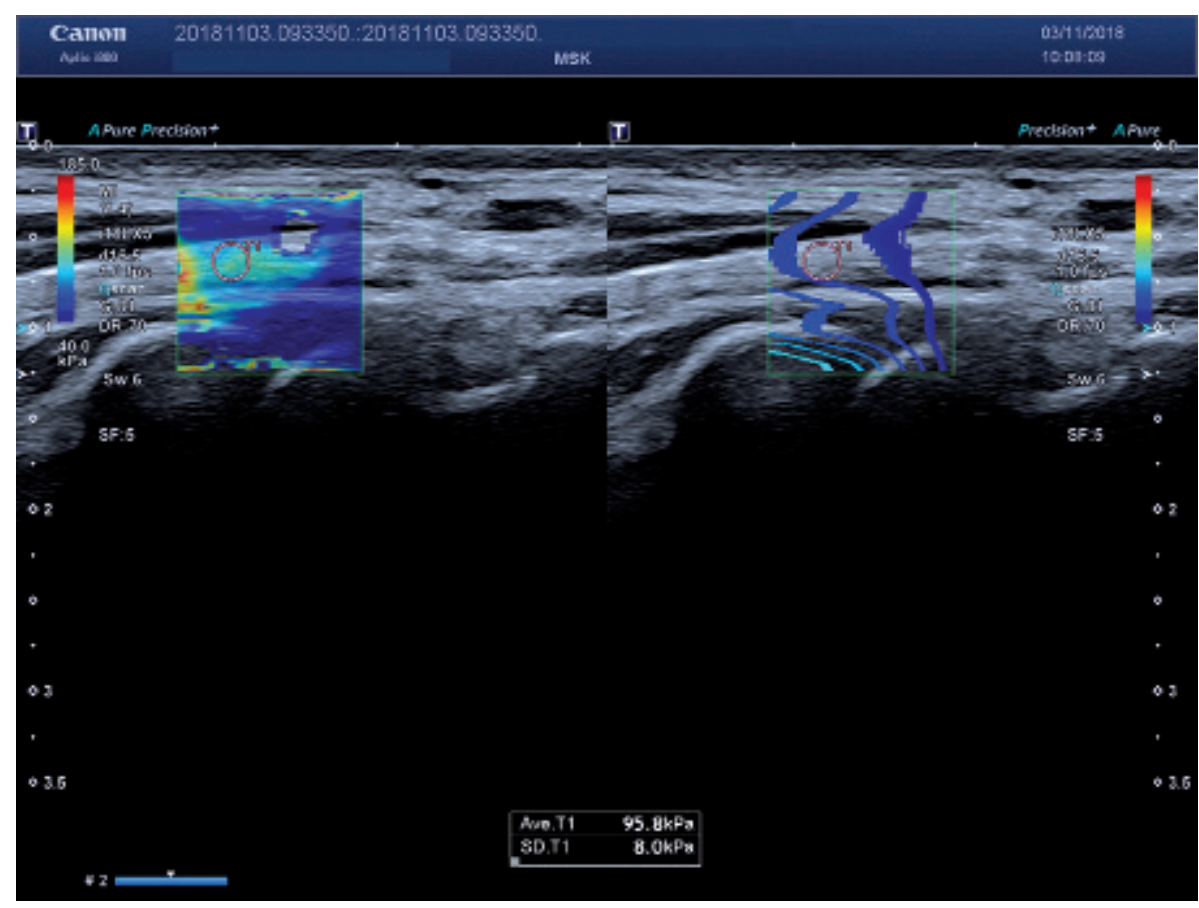

Figure 2. Shear wave elastography image of ulnar nerve proximally to Guyon's canal in a 40-year-old woman with ulnar neuropathy confirmed in nerve conduction studies

researchers (JZ and KZ) independently screened the titles and abstracts to identify the potentially eligible studies. Finally, studies that were potentially eligible were selected for full-text review. Any disagreement was resolved by mutual consent after discussion. The characteristics recorded for each study included authors' names, publication year, country of origin, publication year, number of patients and controls, elastographic method used, outcome measures along with their values, and whenever available, parameters of diagnostic accuracy and reproducibility measures.

A total of 14 studies satisfying the criteria of this review were identified (Table 1). Published data on the applicability of ultrasound elastography to the evaluation of peripheral neuropathies are presented separately for six different indications. Furthermore, potential limitations of this diagnostic modality are discussed along with directions of future research.

\section{Carpal tunnel syndrome}

Carpal tunnel syndrome (CTS) is the most common compression neuropathy of the upper extremities, resulting from entrapment of the median nerve in the carpal tunnel at the wrist. Currently, the gold standard in the evaluation of CTS is NCS, but its invasiveness and relatively high false-negative rate (up to $30 \%$ ) have led to research on other less invasive and more convenient diagnostic options $[2,3,10]$. One such method is ultrasound examination with the assessment of median nerve morphology and function and the determination of CSA at various levels of the carpal tunnel, mostly at the inlet, mid-tunnel, and outlet [11]. While multiple studies demonstrated that the ultrasonographically determined CSA of the median nerve was markedly higher in CTS patients than in healthy controls, conventional ultrasound was also shown to suffer from a lack of standardisation. As a result, the range of proposed cut-off values for CSA measurements is quite wide: from $6.5 \mathrm{~mm}^{2}$ to $15 \mathrm{~mm}^{2}$. This contributed to substantial heterogeneity of sensitivity (57-97.9\%) and specificity (51-100\%) of ultrasonographically determined CSA in the diagnostics of CTS [7,12-18]. To reduce the discrepancies in the CSA measurements, comparison of the cross-sectional area of the median nerve within the carpal tunnel to an unaffected site, such as the forearm, has been proposed, and the swelling ratio has been suggested as a promising method, but the accuracy of this method is still unclear $[19,20]$.

The results of a few published studies suggest that the drawbacks of conventional ultrasonography can be overcome with ultrasound elastography. The studies, involving either SE or SWE, demonstrated unequivocally that peripheral nerves in patients with CTS become stiffer than in healthy persons. In an SE-based study carried out by Orman et al. [21] in 41 CTS patients (74 wrists) and 24 asymptomatic controls (45 wrists), mean tissue strain in the former group was significantly lower than in the latter $(0.094 \pm 0.045$ vs. $0.145 \pm 0.068)$, which implied that the affected median nerves were stiffer than the normal ones. In the same study, a cut-off strain ratio of 0.0635 was shown to be the most sensitive (sensitivity $88 \%$, specificity $45 \%$ ) and a cut-off of 0.19 the most specific (sensitivity $65 \%$, specificity $88 \%$ ) in distinguishing between persons with CTS and without [21]. In another SE study conducted 
Table 1. Published studies analysing the applicability of ultrasound elastography to the evaluation of peripheral neuropathies

\begin{tabular}{|c|c|c|c|c|}
\hline Authors & Indication & $\begin{array}{l}\text { Examined } \\
\text { nerve(s) }\end{array}$ & $\begin{array}{l}\text { Elastographic } \\
\text { method }\end{array}$ & Participants \\
\hline Orman et al. [21] & CTS & Median nerve & SE & $\begin{array}{l}41 \text { patients with CTS (74 nerves) } \\
24 \text { controls ( } 45 \text { nerves) }\end{array}$ \\
\hline Ghajarzadeh et al. [22] & CTS & Median nerve & SE & $\begin{array}{l}31 \text { patients with CTS ( } 60 \text { nerves) } \\
21 \text { controls ( } 44 \text { nerves) }\end{array}$ \\
\hline Tatar et al. [26] & CTS & Median nerve & SE & $\begin{array}{l}19 \text { patients with CTS ( } 35 \text { nerves) } \\
18 \text { controls ( } 36 \text { nerves) }\end{array}$ \\
\hline Kantarci et al. [23] & CTS & Median nerve & SWE & $\begin{array}{l}37 \text { patients with (TS ( } 60 \text { nerves) } \\
18 \text { controls ( } 36 \text { nerves) }\end{array}$ \\
\hline Zhang et al. [24] & CTS & Median nerve & SWE & $\begin{array}{l}49 \text { patients with (TS ( } 72 \text { nerves) } \\
23 \text { controls ( } 46 \text { nerves) }\end{array}$ \\
\hline Paluch et al. [25] & CTS & Median nerve & SWE & $\begin{array}{l}87 \text { nerves with CTS } \\
34 \text { nerves without CTS }\end{array}$ \\
\hline Paluch et al. [29] & UTS & Ulnar nerve & SWE & $\begin{array}{l}46 \text { patients with UTS } \\
39 \text { controls }\end{array}$ \\
\hline Paluch et al. [31] & UNE & Ulnar nerve & SWE & $\begin{array}{l}34 \text { patients with UTS } \\
38 \text { controls }\end{array}$ \\
\hline Ishibashi et al. [41] & DPN & Tibial nerve & SE & $\begin{array}{l}198 \text { patients with type } 2 \text { diabetes mellitus } \\
29 \text { non-diabetic controls }\end{array}$ \\
\hline Dikici et al. [40] & DPN & Tibial nerve & SWE & $\begin{array}{l}20 \text { patients with type } 2 \text { diabetes mellitus and DPN } \\
20 \text { patients with type } 2 \text { diabetes mellitus without DPN } \\
20 \text { non-diabetic controls }\end{array}$ \\
\hline He et al. [43] & DPN & $\begin{array}{l}\text { Tibial nerve } \\
\text { Median nerve }\end{array}$ & SWE & $\begin{array}{l}40 \text { patients with type } 2 \text { diabetes mellitus and DPN } \\
40 \text { patients with type } 2 \text { diabetes mellitus without DPN } \\
40 \text { non-diabetic controls }\end{array}$ \\
\hline Yagci et al. [48] & $\mathrm{CTS}$ in $\mathrm{SSC}$ & Median nerve & SE & $\begin{array}{c}24 \text { patients with SSc without CTS (47 nerves) } \\
27 \text { patients with SSc and CTS (53 nerves) } \\
19 \text { controls ( } 38 \text { nerves) }\end{array}$ \\
\hline Inal et al. [53] & $\begin{array}{l}\text { Optic neuropathy } \\
\text { in BD }\end{array}$ & Optic nerve & $\begin{array}{l}\text { SE } \\
\text { SWE }\end{array}$ & $\begin{array}{l}23 \text { patients with BD (46 nerves) } \\
27 \text { controls ( } 54 \text { nerves) }\end{array}$ \\
\hline Inal et al. [62] & $\begin{array}{l}\text { Optic neuritis } \\
\text { in MS }\end{array}$ & Optic nerve & $\begin{array}{l}\text { SE } \\
\text { SWE }\end{array}$ & $\begin{array}{l}54 \text { patients with MS (107 nerves) } \\
59 \text { controls ( } 118 \text { nerves) }\end{array}$ \\
\hline
\end{tabular}

BD - Behçet's disease, CTS - carpal tunnel syndrome, DPN - diabetic peripheral neuropathy, MS - multiple sclerosis, SE - strain elastography, SSC - systemic sclerosis, SWE - shear wave elastography, UTS - ulnar tunnel syndrome, UNE - ulnar neuropathy of the elbow

by Ghajarzadeh et al. [22], the ratio of total red pixels to total pixels in elastographic images of the median nerve turned out to be significantly higher in healthy controls ( $n=21,44$ nerves) than in individuals with CTS $(n=31$, 60 nerves), which also suggests that the affected nerves are less elastic than the normal ones.

The abovementioned findings are consistent with the results of an SWE-based study carried out by Kantarci et al. [23] in 37 patients (60 wrists) with a definitive diagnosis of CTS and 18 healthy controls (36 wrists). Median nerve stiffness at the carpal tunnel inlet in the CTS group turned out to be significantly higher than in the controls (66.7 kPa vs. $32.0 \mathrm{kPa}$ ), and a $40.4-\mathrm{kPa}$ cut-off value on SWE provided $93.3 \%$ sensitivity, $88.9 \%$ specificity, $93.3 \%$ PPV, $88.9 \%$ NPV, and $91.7 \%$ accuracy in the diagnosis of carpal tunnel syndrome [23]. Similar findings were also reported by Zhang et al. [24]. Those authors used SWE, but instead of the Young modulus values expressed in $\mathrm{kPa}$, they measured shear wave velocity (SWV; $\mathrm{m} / \mathrm{s}$ ) at the carpal tunnel inlet using Virtual Touch tissue imaging quantification. Median nerve SWV in the CTS group ( $n=49,72$ wrists) was significantly higher than in the healthy controls ( $n=23,46$ wrists) $(3.857 \mathrm{~m} / \mathrm{s}$ vs. $2.542 \mathrm{~m} / \mathrm{s}$ ) [24]. Finally, Paluch et al. [25] used SWE to determine the median nerve stiffness in 87 wrists with confirmed CTS and 34 without. Similar to the studies mentioned above, patients with CTS presented with significantly higher median stiffness of the median nerve at the wrist than the controls (100 kPa vs. $42 \mathrm{kPa}$ ), with a cut-off value of $79 \mathrm{kPa}$ providing $96.6 \%$ sensitivity, $100 \%$ 
specificity and positive predictive value (PPV), and 91.9\% negative predictive value (NPV) in distinguishing between the two groups. However, considering the substantial variance in the results of healthy controls (range $24-76 \mathrm{kPa}$ ), the authors proposed to diagnose CTS based on the wristto-forearm ratio of median nerve stiffness, rather than on the basis of the absolute stiffness of the nerve. Median values of the two stiffness ratios obtained by dividing median nerve stiffness at the level of the proximal carpal row and $12 \mathrm{~cm}$ or $20 \mathrm{~cm}$ proximally on the forearm, respectively, were significantly higher in CTS patients than in the controls (2.1 vs. 1.0, regardless of the location), and the cut-off values of 1.483 and 1.508 , respectively, provided the same, excellent accuracy in distinguishing between patients with CTS and without (97.7\% sensitivity, 100\% specificity and PPV, 94.4\% NPV) [25].

While the abovementioned data demonstrate clearly that ultrasound elastography, especially SWE, is suitable for the detection of CTS, less obvious is the link between the median nerve stiffness and severity of the disease. In the SE study conducted by Ghajarzadeh et al. [22], mean total blue pixel number and total blue pixel to total pixel index values increased significantly with advancing CTS severity, but decreased in cases with the most severe form of the disease. According to the authors of this study, this could be associated with axonal loss in more advanced cases [22]. In another SE-based study, Tatar et al. [26] compared median nerve strain ratio at the wrist in 18 healthy volunteers ( 36 wrists) and 19 patients with mild (15 wrists) and moderate to severe CTS (20 wrists). While mean strain ratios in both CTS groups were significantly higher than in the controls $(3.15 \pm 0.09$ in mild CTS and 3.04 \pm 0.96 in moderate-severe CTS vs. $1.85 \pm 0.65$ in the controls), the strain ratio did not change significantly depending on CTS severity [26]. Similarly, Paluch et al. [25] claimed on the lack of statistically significant correlation between the median nerve stiffness determined by means of SWE and CTS severity, although they did not provide the exact numbers supporting this observation. The results of the two studies mentioned above remain in opposition to the findings reported by Kantarci et al. [23], according to whom median nerve stiffness in patients with severe or extremely severe CTS turned out to be significantly higher than in those with mild or moderate disease $(101.4 \mathrm{kPa}$ vs. $55.1 \mathrm{kPa})$.

To summarise, the results of available studies suggest that patients with even mild CTS can be accurately identified using ultrasound elastography, preferably SWE. However, this review of literature demonstrated also substantial heterogeneity of median nerve stiffness in both CTS patients and healthy controls. This implies that aside from the neuropathic process, the stiffness of the median nerve can also be influenced by other factors. Indeed, some experimental data suggest that the results of SWE might be modulated by carpal tunnel pressure. Using the Achilles tendon model, Wang et al. [27] demonstrated that both the absolute SWV and the speed difference between the inside and outside tunnel increased linearly with the tunnel pressure. Furthermore, increased carpal tunnel pressure in CTS was shown to affect blood circulation within the median nerve, and long-term oedema of the nerve is known to trigger fibroblast invasion, resultant accumulation of scar tissue inside the nerve, and an increase in its stiffness [28]. Future studies should verify if and how these experimental data translate onto real-life clinical practice.

\section{Other entrapment neuropathies}

The usefulness of ultrasound elastography in the evaluation of peripheral nerve stiffness in patients with CTS was recently confirmed in two studies analysing other types of entrapment neuropathies.

Paluch et al. [29] analysed the applicability of SWE in ulnar tunnel syndrome (UTS), a compressive neuropathy of the ulnar nerve at the level of Guyon's canal [30]. The study included 46 patients with electromyographically confirmed UTS and 39 healthy controls. Mean SWE at Guyon's canal turned out to be significantly higher in UTS patients than in the controls (99.41 kPa vs. $49.08 \mathrm{kPa}$ ), and the stiffness of $80 \mathrm{kPa}$ or higher, as well as the ratios of ulnar nerve stiffness at Guyon's canal to its stiffness at distal or proximal forearm equal to 1.5 provided an excellent, $100 \%$ sensitivity, specificity, PPV, and NPV in distinguishing between patients with UTS and without [29].

In another study conducted by the same group [31], the SWE stiffness of the ulnar nerve at the cubital tunnel in 34 patients with ulnar neuropathy of the elbow (UNE), the second most common entrapment neuropathy caused by compression of the ulnar nerve at the elbow or its direct proximity [32], was compared with the stiffness of this nerve in 38 healthy controls. Mean SWE of the ulnar nerve in the cubital tunnel was significantly higher in the patient group than in the controls $(96.38 \pm 9.62 \mathrm{kPa}$ vs. $33.08 \pm 10.13 \mathrm{kPa}$ ), and so were the median ratios of the nerve stiffness in the cubital tunnel to its stiffness at the distal arm (2.8 vs. 1.0$)$ and mid-arm (2.7 vs. 1.0). A cut-off value of ulnar nerve stiffness of $61 \mathrm{kPa}$, as well as the ulnar nerve stiffness ratios at distal arm and mid-arm of 1.68 and 1.75 , respectively, provided an excellent $100 \%$ sensitivity, specificity, PPV, and NPV in distinguishing between patients with UNE and without [31].

\section{Diabetic peripheral neuropathy}

Similar to CTS and other entrapment neuropathies, NCS can detect diabetic peripheral neuropathy (DPN) and are considered a gold standard in this indication. However, as already mentioned above, they are time-consuming and invasive; furthermore, the results of NCS in DPN were shown to be biased by skin temperature and humidity [33]. Finally, the latent phase of DPN development is long, 
up to 4-7 years, and hence we need a diagnostic method capable of predicting DPN before functional anomalies emerge on NCS.

Technological progress has opened perspectives for the application of conventional grey-scale ultrasound in the evaluation of patients with suspected DPN. Like in CTS, ultrasound can provide information about the CSA, echogenicity, and inner structure of nerves affected by DPN. However, the results of previous studies analysing the applicability of grey-scale ultrasound for the measurement of CSA in DPN are inconclusive. While some of those studies demonstrated that DPN was associated with an increase in CSA [34-36], others did not find differences in the cross-sectional areas of affected and unaffected nerves [37], or even showed a decrease in the dimensions of the affected nerves [38,39]. Furthermore, a few authors observed that the CSA of peripheral nerves varied depending on the type of the diabetes and its severity. Dikici et al. [40] reported that the CSA of anterior tibial nerve in type 2 diabetic patients with symptomatic neuropathy was different than in healthy controls but not in diabetic patients without the evidence of neuropathy. According to Ishibashi et al. [41], the CSA of tibial nerve in patients with type 2 diabetes mellitus increased with the severity of neuropathy. Breiner et al. [42] demonstrated that type 1 diabetes was associated with an increase in mean peripheral nerve thickness, but the CSA of the affected nerves was smaller than in patients with type 2 diabetes. Finally, in the study conducted by $\mathrm{He}$ et al. [43], CSA of the tibial nerve in patients with DPN was significantly higher than in diabetic patients without the neuropathy and healthy controls, whereas the CTS values for median nerve did not differ significantly between the study groups. According to the authors of this study, the lack of statistically significant between-group differences might be associated with the fact that CSA was determined at the mid-arm where the median nerve is unlikely to be compressed [43]. Regardless of the reason, published evidence shows clearly that the diagnosis of DPN solely on the basis of the CSA can be a subject of considerable bias.

The results of elastographic studies, whether with SE or SWE, are more straightforward. Ishibashi et al. [41] used SE to determine the elasticity of tibial nerves $3 \mathrm{~cm}$ proximal to the medial malleolus in 198 patients with type 2 diabetes mellitus and 29 age- and sex-matched non-diabetic controls. The elasticity, expressed as the elasticity ratio, turned out to be significantly higher in healthy controls $(0.760 \pm 0.0235)$ and to decrease significantly with the severity of diabetic peripheral neuropathy $(0.655 \pm$ 0.0146 vs. $0.542 \pm 0.0144$ vs. $0.475 \pm 0.0193$ vs. $0.414 \pm$ 0.0176 for stages I to VI, respectively). The cut-off value of elasticity of the tibial nerve that suggested the presence of neuropathy was 0.558 and provided $86 \%$ sensitivity and $62 \%$ specificity; these parameters of diagnostic accuracy were significantly higher than in the case of CSA determined by means of conventional ultrasound [41].
The results obtained with SE are consistent with more recent SWE findings. Dikici et al. [40] used SWE to determine the stiffness of tibial nerves in patients with type 2 diabetes mellitus complicated by diabetic peripheral neuropathy $(n=20)$, diabetic patients without neuropathy $(n=20)$, and non-diabetic controls $(n=20)$. The tibial nerve was examined $4 \mathrm{~cm}$ proximal to the medial malleolus. The nerve in patients with established neuropathy proved to be significantly stiffer than in both non-neuropathy diabetic patients and healthy controls (right tibial nerve: $79.5 \pm 25.7$ $\mathrm{kPa}$ vs. $45.6 \pm 16.2 \mathrm{kPa}$ vs. $30.3 \pm 7.6 \mathrm{kPa}$; left tibial nerve: $77.0 \pm 25.3 \mathrm{kPa}$ vs. $36.2 \pm 11.5 \mathrm{kPa}$ vs. $31.3 \pm 11.6 \mathrm{kPa}$ ); furthermore, a significant difference in the right but not left tibial nerve stiffness was found between the latter two groups. A cut-off value of $51.0 \mathrm{kPa}$ provided $90 \%$ sensitivity and $85 \%$ specificity in the detection of diabetic peripheral neuropathy [40]. Finally, He et al. [43] determined SWE stiffness of tibial and median nerve in 40 patients with type 2 diabetes and DPN, 40 diabetic patients without DPN, and 40 healthy controls. The tibial nerve was examined $3 \mathrm{~cm}$ above the medial malleolus and the median nerve at the midpoint of the forearm. The study demonstrated that regardless of the body side, patients with DPN presented with significantly lower SWV in both tibial (left side: 3.67 $\pm 0.47 \mathrm{~m} / \mathrm{s}$ vs. $3.70 \pm 0.49 \mathrm{~m} / \mathrm{s}$ vs. $4.48 \pm 0.59 \mathrm{~m} / \mathrm{s}$; right side: $3.64 \pm 0.49 \mathrm{~m} / \mathrm{s}$ vs. $3.71 \pm 0.52 \mathrm{~m} / \mathrm{s}$ vs. $4.63 \pm 0.54 \mathrm{~m} / \mathrm{s})$ and median nerve (left side: $3.51 \pm 0.57 \mathrm{~m} / \mathrm{s}$ vs. $3.54 \pm 0.60$ $\mathrm{m} / \mathrm{s}$ vs. $4.41 \pm 0.76 \mathrm{~m} / \mathrm{s}$, right side: $3.54 \pm 0.52 \mathrm{~m} / \mathrm{s}$ vs. 3.57 $\pm 0.55 \mathrm{~m} / \mathrm{s}$ vs. $4.40 \pm 0.62 \mathrm{~m} / \mathrm{s}$ ) than the other two groups. The optimal cut-off values for SWE of the tibial nerve and median nerve in the diagnosis of DPN were $4.11 \mathrm{~m} / \mathrm{s}$ and $4.06 \mathrm{~m} / \mathrm{s}$, respectively, with a good sensitivity $(81.3 \%$ and $80 \%$, respectively) and specificity $(88.7 \%$ and $85 \%$, respectively). Interestingly, while the CSA of both left and right tibial nerve was significantly higher in DPN patients, no statistically significant between-group differences were observed in median nerve CSA [43].

In summary, published evidence suggests that ultrasound elastography, especially SWE, might find application as a diagnostic test in DPN. In all of the studies mentioned above, patients with DPN presented with significantly higher peripheral nerve stiffness than healthy controls. Furthermore, in two of these studies [40,43], increased stiffness of the examined nerves was also observed in diabetic patients without clinical evidence of DPN, which implies that elastography could be not only a confirmatory but also a screening test for this complication. However, diagnostic accuracy of elastographic parameters in the detection of DPN seems to be relatively low, no greater than $90 \%$, and hence, unless standardised, this method does not seem suitable as a standalone diagnostic test. Furthermore, it needs to be stressed that DPN affects multiple nerves, and thus the fact that a given patient presents with normal stiffness of one of the most commonly examined nerves does not mean that the morphology of the others is not altered. 


\section{Peripheral neuropathy in systemic sclerosis}

In some forms of systemic sclerosis (SSc), an autoimmune condition of unknown aetiology associated with enhanced deposition of collagen in connective tissue, fibrotic lesions may develop in locations other than the skin [44]. Thickening of connective tissue around or within a peripheral nerve may lead to neuropathy, a rare albeit serious complication of SSc. According to literature, the most frequent forms of peripheral neuropathy in SSc are trigeminal neuropathy, peripheral sensorimotor neuropathy, and CTS [45]. It is still unclear whether these conditions develop in a typical mechanism of compression neuropathy or are also associated with the deposition of collagen within the nerve. The aim of a few previous studies was to address this issue based on ultrasonographic and/or elastographic examination of the median nerve in patients who developed CTS secondarily to SSc.

The evidence from these studies suggests that CTS occurring concomitantly to SSc is also associated with ultrasonographically-detectable morphological alterations of the median nerve. Specifically, patients with SSc presented with larger CSA, higher transverse diameters, and higher flattening ratios of the median nerve than healthy controls [46] and had lower ratio of fascicular to non-fascicular structures within the nerve [47]. These findings are consistent with the ultrasonographic observations of patients with CTS of other aetiology, suggesting that peripheral neuropathy in SSc might develop in a compression mechanism.

However, the results of a study analysing the stiffness of median nerve in SSc (to the best of our knowledge, the only one conducted to date) imply that systemic sclerosis might also affect the function of the median nerve directly. Yagci et al. [48] used SE to examine 24 patients (47 median nerves) with SSc but without any evidence of CTS from nerve conduction studies, 27 patients (53 nerves) with idiopathic CTS confirmed on NCS, and 19 healthy volunteers ( 38 nerves). While SSc patients and healthy controls presented with significantly lower CSA of the median nerve than individuals with CTS, the stiffness of the nerve, expressed as elastic ratios at pisiform and forearm levels was significantly higher in SSc than in other groups (pisiform level: $3.02 \pm 2.56$ in SSc group vs. $2.19 \pm 0.93$ in CTS group vs. $1.63 \pm 1.15$ in the controls; forearm level: $3.21 \pm 1.81$ vs. $2.29 \pm 1.39$ vs. $1.77 \pm 1.21$, respectively). According to the authors of the study, the fact that median nerves in patients with SSc were stiffer than in persons with CTS suggests that also thickening of connective tissue within the nerve might play a role in the development of peripheral neuropathy secondary to systemic sclerosis [48].

Unfortunately, based on the results of this single study, we cannot conclude unequivocally that the aetiology of CTA (and perhaps also other peripheral neuropathies) in SSc is disease-specific or multifactorial, especially because, at the time of the elastographic evaluation, SSc patients participating in the study conducted by Yagci et al. [48] did not show any abnormalities in the results of NCS. Hence, a longitudinal elastographic study, preferably involving SWE, is needed to formulate any ultimate conclusions about the aetiology of peripheral neuropathy in SSc. Nevertheless, even based on the limited available evidence, ultrasound elastography seems to be a valuable diagnostic instrument in this indication.

\section{Optic neuropathy in Behçet's disease}

Behçet's disease (BD) is a chronic autoimmune disease with heterogeneous clinical manifestation, being a consequence of multisystem vasculitis of unknown origin [49]. BD may also produce multiple ocular complications, such as relapsing, remitting panuveitis, retinal vasculitis, retinitis, retinal haemorrhage, macular oedema, and retinal vascular occlusion and necrosis [50,51]. Optic neuropathy in $\mathrm{BD}$ may be a consequence of the occlusion of small supplying vessels and ascending inflammation spreading from the uveal tract $[49,50,52]$. In late stages, the neuropathy is associated with axonal degeneration and fibrosis [50,52]; these processes might contribute to greater stiffness of the optic nerve, analogously to the other peripheral neuropathies described above.

To the best of our knowledge, this hypothesis was verified in only one study. Inal et al. [53] examined 46 optic nerves from 23 patients with $\mathrm{BD}$ and 54 optic nerves from 27 healthy volunteers using conventional ultrasound, SE, and SWE. The study demonstrated that mean thickness of the optic nerve in BD patients was significantly lower than in the controls ( $4.86 \mathrm{~mm}$ vs. $4.97 \mathrm{~mm}$ ). Furthermore, the authors found a statistically significant difference in the SE elasticity patterns in BD patients and healthy volunteers. Most optic nerves in healthy controls represented type 3 (intermediate tissue, green pixels, $51.8 \%$ ) or type 2 (hard tissue, blue-green pixels, $40.7 \%$ ) and rarely showed the characteristics of type 1 (hardest tissue, blue pixels, $7.5 \%$ ). In contrast, the latter type (43.5\%), as well as type 2 (52.2\%), were the most abundant elasticity patterns in $\mathrm{BD}$ patients. Moreover, BD patients presented with significantly higher SWE stiffness of the optic nerve than the controls (32.1 \pm $13.9 \mathrm{kPa}$ vs. $11.5 \pm 5.6 \mathrm{kPa})$. A cut-off value of $16.5 \mathrm{kPa}$ provided very good accuracy in distinguishing between patients with $\mathrm{BD}$ and without, with sensitivity and specificity of $87 \%, 85.1 \% \mathrm{PPV}$, and $88.7 \% \mathrm{NPV}$. The group of patients with BD included seven persons (14 eyes) with uveitis. Mean SWE stiffness in this group was not significantly higher than in other patients with $\mathrm{BD}(36.4 \mathrm{kPa}$ vs. $30.95 \mathrm{kPa})$ [53].

Taken altogether, these findings imply that an increase in optic nerve stiffness can be detected at early stages of $\mathrm{BD}$ and might predict ocular complications associated with this condition. If these clinically attractive observations were confirmed in a larger longitudinal study, SWE 
could play a key role in the secondary prevention of permanent loss of vision due to $\mathrm{BD}$.

\section{Optic neuropathy in multiple sclerosis}

Multiple sclerosis (MS) is a chronic inflammatory neurodegenerative disease affecting the central nervous system $[54,55]$ and associated with demyelination and iron-related abnormalities [56,57]. While impaired vision, one of the most common symptoms of MS, may be associated with involvement of the visual system at any anatomical site, its best known pathomechanism is inflammatory demyelinating optic neuritis $[58,59]$. This condition can be detected with conventional and non-conventional MRI techniques, such as diffusion tensor imaging, diffusion-weighted imaging, and magnetisation transfer imaging [60,61], but they are time consuming, costly, and less available.

In the search for an alternative diagnostic method, Inal et al. [62] examined 107 optic nerves from $54 \mathrm{MS}$ patients (among them 27 nerves with previous optic neuritis diagnosed based on medical history and clinical findings) and 118 optic nerves from 59 healthy controls, using SE and SWE. Based on the results of SE, optic nerves were classified into three elasticity types: type 1 , the hardest (with predominance of blue pixels); type 2 , hard (with predominance of blue/green pixels; and type 3, intermediate (with predominance of green pixels). The study demonstrated significant differences in the distribution of these elasticity types in MS patients in healthy controls. While in all controls, optic nerves represented type (61.9\%) or type 2 (38.1\%), the vast majority of MS patients (88\%) presented with type 2 optic nerves, and type 3 was found in no more than $6.5 \%$ of the cases. Also, the SWE stiffness of the optic nerve differed significantly between the study groups, with significantly higher values found in MS patients than in the controls $(33.87 \pm 11.64 \mathrm{kPa}$ vs. $10.381 \pm 3.48 \mathrm{kPa})$. A cutoff value of $18.3 \mathrm{kPa}$ was shown to provide very high sensitivity, specificity, PPV, and NPV (95.3\%, 97.5\%, 97.2\%, and $95.8 \%$, respectively) in distinguishing between MS patients and healthy controls. Importantly, no significant differences in SWE stiffness were observed when optic nerves of MS patients were stratified into those with the history of previous neuritis and without $(33.87,11.6$ vs. $33.88,11.33)$ [62] .

While these findings undoubtedly need to be verified in a larger series of patients, the evidence from this single study suggests that ultrasound elastography, whether SE or SWE, could be used to detect MS-related anomalies in the optic nerve. Considering the lack of statistically significant differences in the SWE stiffness of the optic nerve in patients with a history of neuritis and without, these anomalies might be detected even at early, asymptomatic stages.

\section{Technical aspects to be addressed}

While some of the studies reviewed above used SE to determine the peripheral nerve stiffness, SWE seems to be more applicable in this indication. First, the result of SWE is $100 \%$ quantitative, unlike the qualitative or semi-quantitative outcome of SE. Second, SWE is less operator dependent because the examination does not require controlled compression of tissue with a transducer, hence eliminating a source of potential bias. These two factors contributed to very good or excellent reproducibility of SWE results in the evaluation of peripheral nerve stiffness. According to Kantarci et al. [23], the inter-observer agreement rates in the measurement of median nerve stiffness in CTS patients and healthy controls were excellent: 0.848 and 0.809 , respectively. Even higher consistency of SWE measurements was reported by He et al. [43] in the evaluation of median and tibial nerve stiffness in patients with DPN, diabetic patients without neuropathy, and healthy controls; the inter- and intra-observer agreement rates in this study were 0.958 and 0.960 , respectively. According to Bortolotto et al. [9], the inter-observer agreement in the evaluation of median nerve stiffness in healthy volunteers was fair to moderate (intraclass correlation coefficients, ICC between 0.38 and 0.44 , depending on the examined site), but it needs to be emphasised that the study involved one expert and one inexperienced operator, with six years and six months of ultrasound experience, respectively.

Although the results presented in this review are without doubt promising, some issues still need to be addressed before ultrasound elastography can be routinely used in the evaluation of peripheral neuropathies. One of the key questions is the identification of normal values for peripheral nerve stiffness, because the evidence from most published studies suggests that even in healthy persons, the results of elastography can be quite heterogeneous. For example, mean median nerve stiffness at the wrist in CTSfree controls included in the study conducted by Paluch et al. [25] was higher than in CTS-free volunteers examined by Kantarci et al. [23] (43.6 kPa vs. $32 \mathrm{kPa}$, respectively). Furthermore, the range of normal values in the former study was highly heterogeneous $(24-76 \mathrm{kPa})$ and partially overlapped with those found in CTS patients $(35-135 \mathrm{kPa})$ [25]. The same refers to the cut-off values distinguishing between the neuropathy patients and healthy controls, which differed substantially from study to study. For example, the cut-off value that distinguished between CTS patients and healthy persons in the study by Paluch et al. [25] was nearly twice as high as the one identified by Kantarci et al. [23] (79 kPa vs. $40.4 \mathrm{kPa}$ ).

Some authors made an attempt to identify the reference values for SWE peripheral nerve stiffness, but these relatively small studies produced quite inconclusive results. For example, Bedewi et al. [63] evaluated the SWE stiffness of C5-C7 nerve roots of the brachial plexus at the interscalene interval in 40 healthy persons, obtaining relatively low mean values: $16.9 \pm 4.9 \mathrm{kPa}$ for $\mathrm{C} 5,15.7 \pm$ $4.3 \mathrm{kPa}$ for $\mathrm{C} 6$, and $16.0 \pm 4.6 \mathrm{kPa}$ for $\mathrm{C} 7$. The same group [64] made an attempt to determine the SWE stiffness of median nerve in 10 patients ( 20 nerves) with no history 
of CTS or other neuropathies. Mean SWE stiffness at the wrist and $10 \mathrm{~cm}$ proximally was $20.78 \pm 5.11$ and 11.43 \pm 6.30 [64], respectively. These values were considerably lower than the results for healthy controls in most SWE studies of the median nerve mentioned in this review. Moreover, they differed from the results obtained by Bortolotto et al. [9] in 36 healthy volunteers with no present or past history of carpal tunnel syndrome, other forearm and wrist pathologies, and medical conditions possibly favouring CTS development. Mean values of median nerve stiffness in this group were $32.26 \pm 18.6 \mathrm{kPa}$ within the carpal tunnel, $22.20 \pm 9.84 \mathrm{kPa}$ at the tunnel inlet, and $7.62 \pm 7.38 \mathrm{kPa}$ in the forearm [9].

As emphasised by Paluch et al. [65], the discrepancies in the results of previous studies of healthy controls might be explained by methodological differences (discussed in detail below) and/or the influence of some biological confounders. Relationships between peripheral nerve stiffness and patient-related characteristics were analysed by only a few authors, and the results of these studies are inconclusive. In the study of Ishibashi et al. [41], the elasticity of tibial nerve in type 2 diabetic patients with DPN and healthy controls correlated inversely with age. However, Yagci et al. [48] found no significant correlations between the SWE stiffness of the optic nerve and age of either SSc patients or healthy controls. Similarly, no significant correlations between the optic nerve stiffness and age were found in patients with MS and healthy controls examined by Inal et al. [62].

Aside from the severity of peripheral neuropathy and its underlying condition (discussed in detail in previous chapters) and the potential biological confounders mentioned above, another source of variance in the results of elastographic studies can be methodological differences and some technological drawbacks. Available evidence suggests that the SWE stiffness of peripheral nerves can change depending on the plane on which the measurements were taken [65]. This issue was addressed well in the study conducted by Aslan et al. [39], who examined median and posterior tibial nerve stiffness in 25 adolescents with type 1 diabetes mellitus without DPN and 32 healthy volunteers. The SWE measurements were taken by two observers on the transverse and longitudinal axes. Interobserver agreement for the shear modulus, elastic modulus, and SVW were lower on the transverse axis (0.210-0.633) than on the longitudinal axis (0.682-0.748). Based on those findings, the authors concluded that the reliability of the SWE measurements might be jeopardised by the imaging axis, unintentional compression on the transverse axis, and small microarchitectural differences through the nerve, and recommended longitudinal-axis SWE as more appropriate, proper, practical, and reproducible for peripheral nerves [39].

Another technological aspect that needs to be addressed during the elastographic evaluation of peripheral nerves is the close proximity of bone surfaces that might act as hardening artefacts [9]. Such artefacts occur when the studied structure is near a hard plane (e.g. bone) that prevents homogeneous propagation of shear wave at depth and contributes to local stress inhomogeneity [66]. To the best of our knowledge, the impact of these artefacts on the SWE measurements of peripheral nerve stiffness has not been analysed thus far.

\section{Conclusions}

The evidence presented above shows clearly that ultrasound elastography can accurately diagnose many types of peripheral neuropathies, not infrequently at the stages at which the condition is still asymptomatic. However, we still do not know whether elastographic changes within the nerves precede functional anomalies detectable on nerve conduction studies. Also, relatively little is known about the relationship between the stiffness of peripheral nerves and the severity of peripheral neuropathy and its underlying condition, although the results of some studies suggest that such a link might exist. These two issues need to be addressed in large-scale studies, preferably longitudinal ones.

While based on the reproducibility data, SWE seems to be superior to SE, we still need to identify the sources of heterogeneity in the peripheral nerve stiffness in healthy persons. This information, along with the results of population-based studies of healthy persons, should be used to identify the sets of reference values for each peripheral nerve that is considered important from a clinical perspective. Finally, potential confounding effect of hardening artefacts, such as bones, on the stiffness of peripheral nerves needs to be verified, and some technological countermeasures should be introduced if necessary.

If all of the above issues are addressed satisfactorily, elastographic evaluation of peripheral nerve stiffness might become a reliable, easily accessible, and convenient diagnostic test performed routinely in patients with various peripheral neuropathies.

\section{Disclosure}

The authors declare no conflicts of interest.

\section{References}

1. Watson JC, Dyck PJ. Peripheral neuropathy: a practical approach to diagnosis and symptom management. Mayo Clin Proc 2015; 90: 940-951.

2. Witt JC, Hentz JG, Stevens JC. Carpal tunnel syndrome with normal nerve conduction studies. Muscle Nerve 2004; 29: 515-522.

3. Koyuncuoglu HR, Kutluhan S, Yesildag A, et al. The value of ultrasonographic measurement in carpal tunnel syndrome in patients with negative electrodiagnostic tests. Eur J Radiol 2005; 56: 365-369.

4. Andreisek G, Crook DW, Burg D, et al. Peripheral neuropathies of the median, radial, and ulnar nerves: MR imaging features. Radiographics 2006; 26: 1267-1287. 
5. Dyck PJ, Overland CJ, Low PA, et al. Signs and symptoms versus nerve conduction studies to diagnose diabetic sensorimotor polyneuropathy: Cl vs. NPhys trial. Muscle Nerve 2010; 42: 157-164.

6. Klauser AS, Halpern EJ, De Zordo T, et al. Carpal tunnel syndrome assessment with US: value of additional cross-sectional area measurements of the median nerve in patients versus healthy volunteers. Radiology 2009; 250: 171-177.

7. Pastare D, Therimadasamy AK, Lee E, et al. Sonography versus nerve conduction studies in patients referred with a clinical diagnosis of carpal tunnel syndrome. J Clin Ultrasound 2009; 37: 389-393.

8. Cosgrove D, Piscaglia F, Bamber J, et al. EFSUMB guidelines and recommendations on the clinical use of ultrasound elastography. Part 2: Clinical applications. Ultraschall Med 2013; 34: 238-253.

9. Bortolotto C, Turpini E, Felisaz P, et al. Median nerve evaluation by shear wave elastosonography: impact of "bone-proximity" hardening artifacts and inter-observer agreement. J Ultrasound 2017; 20: 293-299.

10. Atroshi I, Gummesson C, Johnsson R, et al. Diagnostic properties of nerve conduction tests in population-based carpal tunnel syndrome. BMC Musculoskelet Disord 2003; 4: 9.

11. Ghasemi-Esfe AR, Morteza A, Khalilzadeh O, et al. Color Doppler ultrasound for evaluation of vasomotor activity in patients with carpal tunnel syndrome. Skeletal Radiol 2012; 41: 281-286.

12. Nakamichi KI, Tachibana S. Enlarged median nerve in idiopathic carpal tunnel syndrome. Muscle Nerve 2000; 23: 1713-1718.

13. El Miedany YM, Aty SA, Ashour S. Ultrasonography versus nerve conduction study in patients with carpal tunnel syndrome: substantive or complementary tests? Rheumatology (Oxford) 2004; 43: 887-895.

14. Wong SM, Griffith JF, Hui AC, et al. Carpal tunnel syndrome: diagnostic usefulness of sonography. Radiology 2004; 232: 93-99.

15. Yesildag A, Kutluhan S, Sengul N, et al. The role of ultrasonographic measurements of the median nerve in the diagnosis of carpal tunnel syndrome. Clin Radiol 2004; 59: 910-915.

16. Ziswiler HR, Reichenbach S, Vögelin E, et al. Diagnostic value of sonography in patients with suspected carpal tunnel syndrome: a prospective study. Arthritis Rheum 2005; 52: 304-311.

17. Wiesler ER, Chloros GD, Cartwright MS, et al. The use of diagnostic ultrasound in carpal tunnel syndrome. J Hand Surg Am 2006; 31 : 726-732.

18. Sernik RA, Abicalaf CA, Pimentel BF, et al. Ultrasound features of carpal tunnel syndrome: a prospective case-control study. Skeletal Radiol 2008; 37: 49-53.

19. Hobson-Webb LD, Massey JM, Juel VC, et al. The ultrasonographic wrist-to-forearm median nerve area ratio in carpal tunnel syndrome. Clin Neurophysiol 2008; 119: 1353-1357.

20. Visser LH, Smidt MH, Lee ML. Diagnostic value of wrist median nerve cross sectional area versus wrist-to-forearm ratio in carpal tunnel syndrome. Clin Neurophysiol 2008; 119: 2898-2899.

21. Orman G, Ozben S, Huseyinoglu N, et al. Ultrasound elastographic evaluation in the diagnosis of carpal tunnel syndrome: initial findings. Ultrasound Med Biol 2013; 39: 1184-1189.

22. Ghajarzadeh M, Dadgostar M, Sarraf P, et al. Application of ultrasound elastography for determining carpal tunnel syndrome severity. Jpn J Radiol 2015; 33: 273-278.
23. Kantarci F, Ustabasioglu FE, Delil S, et al. Median nerve stiffness measurement by shear wave elastography: a potential sonographic method in the diagnosis of carpal tunnel syndrome. Eur Radiol 2014; 24: 434-440.

24. Zhang C, Li M, Jiang J, et al. Diagnostic value of virtual touch tissue imaging quantification for evaluating median nerve stiffness in carpal tunnel syndrome. J Ultrasound Med 2017; 36: 1783-1791.

25. Paluch $€$, Pietruski P, Walecki J, et al. Wrist to forearm ratio as a median nerve shear wave elastography test in carpal tunnel syndrome diagnosis. J Plast Reconstr Aesthet Surg 2018; 71: 1146-1152.

26. Tatar IG, Kurt A, Yavasoglu NG, et al. Carpal tunnel syndrome: elastosonographic strain ratio and cross-sectional area evaluation for the diagnosis and disease severity. Med Ultrason 2016; 18: 305-311.

27. Wang Y, Qiang B, Zhang X, et al. A non-invasive technique for estimating carpal tunnel pressure by measuring shear wave speed in tendon: a feasibility study. J Biomech 2012; 45: 2927-2930.

28. Rempel D, Dahlin L, Lundborg G. Pathophysiology of nerve compression syndromes: response of peripheral nerves to loading. J Bone Joint Surg Am 1999; 81: 1600-1610.

29. Paluch $€$, Noszczyk BH, Walecki J, et al. Shear-wave elastography in the diagnosis of ulnar tunnel syndrome. J Plast Reconstr Aesthet Surg 2018; 71: 1593-1599.

30. Bachoura A, Jacoby SM. Ulnar tunnel syndrome. Orthop Clin North Am 2012; 43: 467-474.

31. Paluch $€$, Noszczyk B, Nitek Ż, et al. Shear-wave elastography: a new potential method to diagnose ulnar neuropathy at the elbow. Eur Radiol 2018; 28: 4932-4939.

32. Assmus H, Antoniadis G, Bischoff C, et al. Cubital tunnel syndrome - a review and management guidelines. Cent Eur Neurosurg 2011; 72: 90-98.

33. Colak A, Kutlay M, Pekkafali Z, et al. Use of sonography in carpal tunnel syndrome surgery. A prospective study. Neurol Med Chir (Tokyo) 2007; 47: 109-115.

34. Kelle B, Evran M, Ballı T, et al. Diabetic peripheral neuropathy: correlation between nerve cross-sectional area on ultrasound and clinical features. J Back Musculoskelet Rehabil 2016; 29: 717-722.

35. Pitarokoili K, Kerasnoudis A, Behrendt V, et al. Facing the diagnostic challenge: nerve ultrasound in diabetic patients with neuropathic symptoms. Muscle Nerve 2016; 54: 18-24.

36. Singh K, Gupta K, Kaur S. High resolution ultrasonography of the tibial nerve in diabetic peripheral neuropathy. J Ultrason 2017; 17 : 246-252.

37. Hobson-Webb LD, Massey JM, et al. Nerve ultrasound in diabetic polyneuropathy: correlation with clinical characteristics and electrodiagnostic testing. Muscle Nerve 2013; 47: 379-384.

38. Riazi S, Bril V, Perkins BA, et al. Can ultrasound of the tibial nerve detect diabetic peripheral neuropathy? A cross-sectional study. Diabetes Care 2012; 35: 2575-2579.

39. Aslan M, Aslan A, Emeksiz HC, et al. Assessment of peripheral nerves with shear wave elastography in type 1 diabetic adolescents without diabetic peripheral neuropathy. J Ultrasound Med 2019; 38 : 1583-1596.

40. Dikici AS, Ustabasioglu FE, Delil S, et al. Evaluation of the tibial nerve with shear-wave elastography: a potential sonographic method for the diagnosis of diabetic peripheral neuropathy. Radiology 2017; 282: 494-501. 
41. Ishibashi F, Taniguchi M, Kojima R, et al. Elasticity of the tibial nerve assessed by sonoelastography was reduced before the development of neuropathy and further deterioration associated with the severity of neuropathy in patients with type 2 diabetes. J Diabetes Investig 2016; 7: 404-412.

42. Breiner A, Qrimli M, Ebadi H, et al. Peripheral nerve high-resolution ultrasound in diabetes. Muscle Nerve 2017; 55: 171-178.

43. He Y, Xiang X, Zhu BH, et al. Shear wave elastography evaluation of the median and tibial nerve in diabetic peripheral neuropathy. Quant Imaging Med Surg 2019; 9: 273-282.

44. Varga JA, Trojanowska M. Fibrosis in systemic sclerosis. Rheum Dis Clin North Am 2008; 34: 115-143.

45. Amaral TN, Peres FA, Lapa AT, et al. Neurologic involvement in scleroderma: a systematic review. Semin Arthritis Rheum 2013; 43: 335-347.

46. Bandinelli F, Kaloudi O, Candelieri A, et al. Early detection of median nerve syndrome at the carpal tunnel with high-resolution 18 $\mathrm{MHz}$ ultrasonography in systemic sclerosis patients. Clin Exp Rheumatol 2010; 28 (5 Suppl 62): S15-S18.

47. Bignotti B, Ghio M, Panico N, et al. High-resolution ultrasound of peripheral nerves in systemic sclerosis: a pilot study of computer-aided quantitative assessment of nerve density. Skeletal Radiol 2015; 44: 1761-1767

48. Yagci I, Kenis-Coskun O, Ozsoy T, et al. Increased stiffness of median nerve in systemic sclerosis. BMC Musculoskelet Disord 2017; 18: 434 .

49. Kidd DP. Optic neuropathy in Behçet's syndrome. J Neurol 2013; 260: 3065-3070.

50. Voros GM, Sandhu SS, Pandit R. Acute optic neuropathy in patients with Behçet's disease. Report of two cases. Ophthalmologica 2006; 220: 400-405

51. Ucar D, Uygunoglu U, Dikkaya F, et al. Retinal nerve fiber layer structure abnormalities in patients with neuro-Behcet's disease. Graefes Arch Clin Exp Ophthalmol 2015; 253: 1181-1185.

52. Cetin EN, Yaylali V, Yildirim C. Isolated optic neuropathy in a case of Behçet's disease. Int Ophthalmol 2011; 31: 153-155.

53. Inal $M$, Tan $S$, Demirkan $S$, et al. Evaluation of optic nerve with strain and shear wave elastography in patients with Behçet's disease and healthy subjects. Ultrasound Med Biol 2017; 43: 13481354.

54. Raz E, Bester M, Sigmund EE, et al. A better characterization of spinal cord damage in multiple sclerosis: a diffusional kurtosis imaging study. AJNR Am J Neuroradiol 2013; 34: 1846-1852.

55. Hojjat SP, Kincal M, Vitorino R, et al. Cortical perfusion alteration in normal-appearing gray matter is most sensitive to disease progression in relapsing-remitting multiple sclerosis. AJNR Am J Neuroradiol 2016; 37: 1454-1461

56. Assaf Y, Chapman J, Ben-Bashat D, et al. White matter changes in multiple sclerosis: correlation of q-space diffusion MRI and 1H MRS. Magn Reson Imaging 2005; 23: 703-710.

57. Chawla S, Kister I, Wuerfel J, et al. Iron and non-iron-related characteristics of multiple sclerosis and neuromyelitis optica lesions at 7T MRI. AJNR Am J Neuroradiol 2016; 37: 1223-1230.

58. Kolbe S, Chapman C, Nguyen T, et al. Optic nerve diffusion changes and atrophy jointly predict visual dysfunction after optic neuritis. Neuroimage 2009; 45: 679-686.
59. Khanna S, Sharma A, Huecker J, et al. Magnetic resonance imaging of optic neuritis in patients with neuromyelitis optica versus multiple sclerosis. J Neuroophthalmol 2012; 32: 216-220

60. Sbardella E, Tona F, Petsas N, et al. DTI measurements in multiple sclerosis: evaluation of brain damage and clinical implications. Mult Scler Int 2013; 2013: 671730. doi: 10.1155/2013/671730

61. Inal M, Unal B, Kala I, et al. ADC evaluation of the corticospinal tract in multiple sclerosis. Acta Neurol Belg 2015; 115: 105-109.

62. Inal $M$, Tan $S$, Yumusak EM, et al. Evaluation of the optic nerve using strain and shear wave elastography in patients with multiple sclerosis and healthy subjects. Med Ultrason 2017; 19: 39-44.

63. Bedewi MA, Nissman D, Aldossary NM, et al. Shear wave elastography of the brachial plexus roots at the interscalene groove. Neurol Res 2018; 40: 805-810.

64. Bedewi MA, Coraci D, Ruggeri F, et al. Shear wave elastography of median nerve at wrist and forearm. Heterogeneity of normative values. J Plast Reconstr Aesthet Surg 2019; 72: 137-171.

65. Paluch L, Pietruski P, Walecki J, et al. Shear wave elastography of median nerve at wrist and forearm. Heterogeneity of normative values: response to authors. J Plast Reconstr Aesthet Surg 2019; 72: 137-171.

66. Bhatia KS, Cho CC, Tong CS, et al. Shear wave elastography of focal salivary gland lesions: preliminary experience in a routine head and neck US clinic. Eur Radiol 2012; 22: 957-965. 